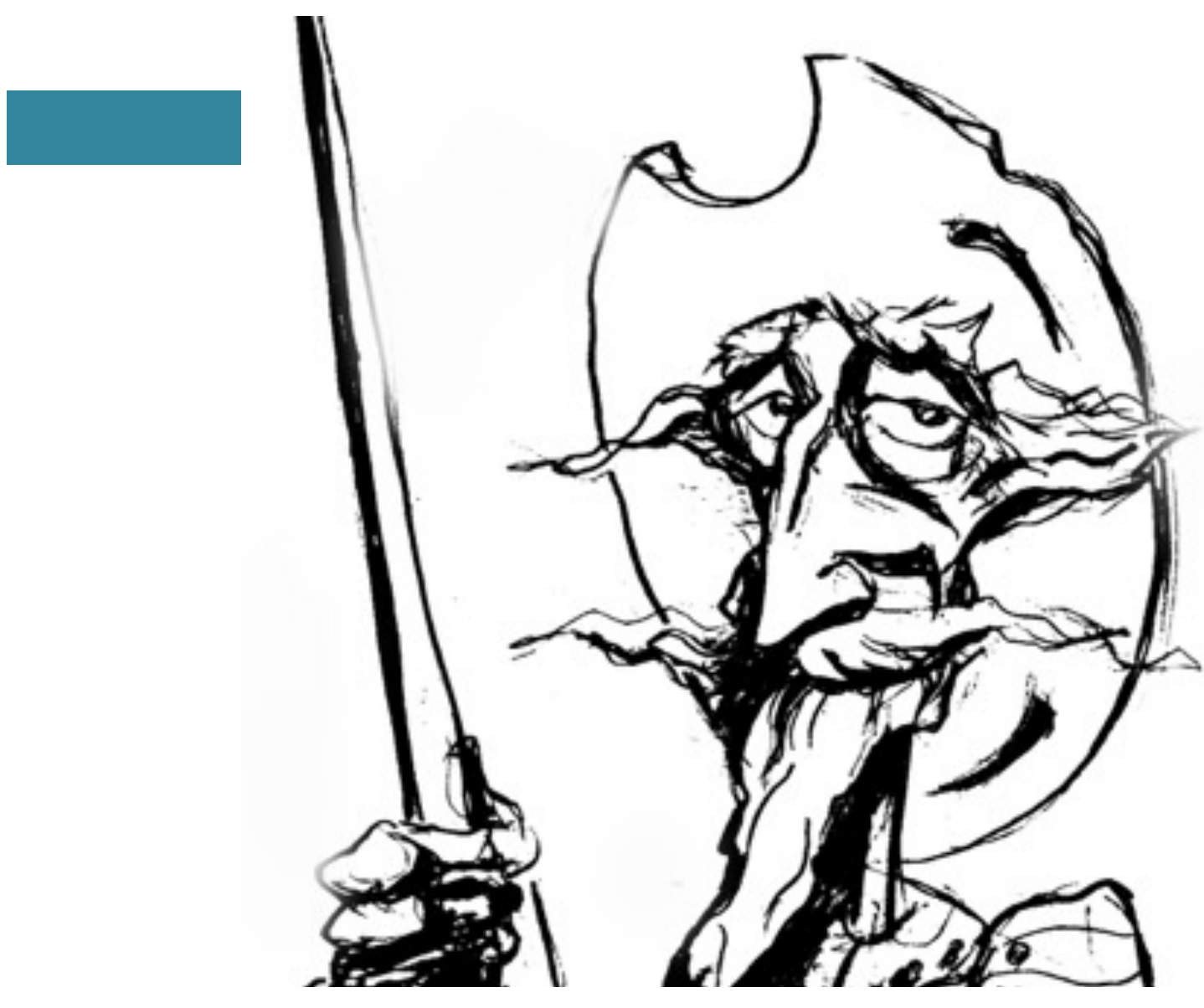

El dialogo continúa. Formas de articulación entre los notables de San Antonio de Areco y el gobierno del Estado de Buenos Aires

[Mariana Canedo] 


\title{
El dialogo continúa. Formas de articulación entre los notables de San Antonio de Areco y el gobierno del Estado de Buenos Aires
}

\author{
The dialogue continues. Forms of engage between the notables of San \\ Antonio de Areco and the government of the Estado de Buenos Aires
}

MARIANA CANEDO

\begin{abstract}
Resumen
El objetivo del trabajo es analizar articulaciones entabladas entre los notables y municipales de los pueblos con el nuevo gobierno del Estado de Buenos Aires. En diálogo con los aportes del libro de Juan Carlos Garavaglia reconstruiremos para el caso de San Antonio de Areco tres situaciones que iluminan dichas relaciones: la construcción de un puente impulsada por vecinos accionistas, las particularidades del presupuesto municipal para 1858, y las tensiones entre jurisdicciones por parte de la municipalidad y el cura párroco, cuya prolongada resolución permitirá vislumbrar cambios más allá del Estado de Buenos Aires en los procesos de negociación y los sujetos intervinientes.
\end{abstract}

Palabras clave

Garavaglia - Areco - notables - municipalidades - Estado de Buenos Aires

\begin{abstract}
The objective is to analyze the relations established between the notables and municipales of the towns with the new government of the Estado de Buenos Aires. In dialogue with the contributions of the book of Juan Carlos Garavaglia, we will reconstruct for the case of San Antonio de Areco three situations that illuminate these relations: the laying of a bridge impelled by vecinos accionistas, the particularities of the municipal budget for 1858, and the tensions between jurisdictions of the municipality and the parish priest, whose prolonged resolution it will allow to glimpse changes beyond the Estado de Buenos Aires in the processes of negotiation and the subjects.
\end{abstract}

\section{Key words}

Garavaglia - Areco - Notables - Municipalities Estado de Buenos Aires

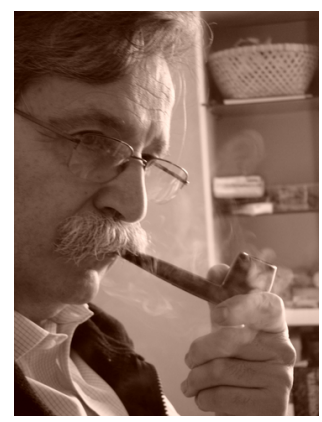

Recibido con pedido de publicación el 20 de agosto de 2017

Aceptado para su publicación el 15 de octubre de 2017

Versión definitiva recibida el 24 de noviembre de 2017

Mariana Canedo, Universidad Nacional de Mar del Plata, Consejo Nacional de Investigaciones Científicas y Técnicas, Grupo Historia Rural Rioplatense, Argentina; e-mail: $\underline{\text { mcanedo@mdp.edu.ar }}$

Esta obra se publica bajo licencia Creative Commons. Atribución-NoComercial-CompartirIgual

(c) EY-NC-SA $\underline{4.0}$ Internacional

Canedo, Mariana "El dialogo continúa. Formas de articulación entre los notables de San Antonio de Areco y el gobierno del Estado de Buenos Aires", Prohistoria, Año XX, núm. 28, Homenaje a Juan Carlos Garavaglia, dic. 2017, pp. 49-67. 
Al conocerse la triste noticia del fallecimiento de Juan Carlos Garavaglia, comenzaron a circular recuerdos, fotos y comentarios por las redes sociales. Varios colegas, además de compartir la tristeza, valoraron su trascendencia a través de los libros. En ese momento, la reflexión me sorprendió pero, pasado casi un año, me encuentro comprobando lo acertada de la misma. El diálogo, de alguna manera, continúa.

Para esta oportunidad, elegí compartir una experiencia de dialogo -entre tantas posibles- que permite uno de los grandes libros de Garavaglia, San Antonio de Areco, 1680-1880. Un pueblo de la campaña, del Antiguo Régimen a la modernidad argentina, con $\mathrm{mi}$ investigación en curso sobre el proceso de municipalización de la campaña de Buenos Aires a mediados del siglo XIX. ${ }^{1}$ El libro es claramente reconocible como hijo de los cambios historiográficos recorridos tras el regreso a la democracia en Argentina y tiene a Pastores y labradores de Buenos Aires. Una historia agraria de la campaña bonaerense, 1700-1830 como uno de sus hermanos mayores. ${ }^{2}$ Dentro de esa familiaridad, con una década de separación entre ellos y casi otra con nuestro presente, los libros permiten distinguir los conocimientos consolidados, las concadenadas problemáticas abordadas, así como la actualización de las agendas de investigación en curso.

Aunque Juan Carlos ubica como destinatarios principales de San Antonio de Areco, 1680-1880 a los vecinos actuales del lugar, el libro está dirigido a varios tipos de lectores y, por lo tanto, se articula en objetivos complejos. En líneas generales podemos decir que busca analizar y comprender la vida de hombres y mujeres, "familias", pertenecientes a distintos sectores sociales, que fueron conformando una comunidad rural en la dinámica jurisdicción de Buenos Aires durante dos siglos (1680-1880). Para hacerlo, apuesta por implicarse en el estudio simultáneo de varias dimensiones de análisis, sin dejar de considerar los ritmos de cada una. Asimismo, jerarquiza en el enfoque el arraigo de los migrantes y nativos al territorio que ellos mismos fueron construyendo y transformando, y no escatima en esfuerzos descriptivos y en incorporar fotografías, mapas y planos para que los lectores podamos comprender mejor cómo era la pampa ondulada, la importancia de sus ríos $\mathrm{y}$, en definitiva, intentemos ubicarnos en San Antonio de Areco y la campaña de Buenos Aires de esos siglos. La propuesta en sí le otorga singularidad al libro y pone en evidencia la osadía de Juan Carlos sostenida por su capacidad, oficio e intensidad laborar. ${ }^{3}$

Garavaglia siempre intentó ir más allá en sus aportes y en este sentido el libro avanza en sus análisis hacia la segunda mitad del siglo XIX. Esta generación de puentes entre historiografías que poco se han influido mutuamente, constituye una práctica que continua siendo escasamente recorrida. Esta se produce en el libro, a mi entender, señalando caminos a transitar con miras a un mejor conocimiento del devenir de esas sociedades.

\footnotetext{
${ }^{1}$ GARAVAGLIA, Juan Carlos San Antonio de Areco, 1680-1880. Un pueblo de la campaña, del Antiguo Régimen a la modernidad argentina, Prohistoria, Rosario, 2009.

${ }^{2}$ GARAVAGLIA, Juan Carlos Pastores y labradores de Buenos Aires. Una historia agraria de la campaña bonaerense, 1700-1830, UNICEN, Universidad Internacional de Andalucía, La Flor, Tandil, 1999.

${ }^{3}$ Una primera experiencia muy atractiva de valoración del libro fue realizada por Darío Barriera, Eduardo Miguez, Roy Hora y Juan Manuel Palacio ni bien salió editado el libro y los respectivos comentarios, incluyendo la presentación de Raúl Fradkin y Jorge Gelman y la respuesta de Juan Carlos a los mismos, fueron publicados en el Anuario del IEHS, núm. 25, Tandil, 2010, pp. 13-41.
} 
Uno de ellos está constituido por los procesos de negociación entre los notables de los pueblos con el estado, y el disciplinamiento o uso de la fuerza hacia los paisanos. $^{4}$

Por mi parte, me encuentro analizando las formas de articulación de los poderes y formas de gobierno locales de los pueblos rurales con el gobierno del Estado de Buenos Aires a mediados del siglo XIX. Particularmente, reconstruyendo la complejidad del proceso de municipalización de la campaña que, por lo menos en la etapa de conformación, permite ser considerado como un caso particular en el contexto iberoamericano. ${ }^{5}$ Constituyó un proceso impulsado de manera prioritaria por el gobierno del Estado de Buenos Aires, que separado de la Confederación Argentina se declaraba soberano; estuvo promovido de forma simultánea a toda la campaña, en una coyuntura compleja con enfrentamientos bélicos; fue extensivo al abarcar a unos cuarenta pueblos mayoritariamente cercanos entre sí, sin requisitos poblacionales, consolidación o distancia entre ellos; y constituyó un proceso relativamente tardío en relación a las prerrogativas establecidas para las noveles municipalidades de campaña. ${ }^{6}$

Consideramos que los esfuerzos efectuados por las alianzas que ejercieron el poder en el Estado de Buenos Aires hacia el proceso de municipalización estuvieron relacionados con la búsqueda de afianzar vínculos políticos en una campaña heterogénea y activa en sus reclamos políticos. Los anhelos del gobierno se presentaban uniformes hacia las municipalidades pero las propuestas y reacciones de las municipalidades y poderes locales se vincularon con las configuraciones propias de cada comunidad política. La mirada de conjunto de las políticas logra mayor sentido al interactuar con los casos y el dialogo con los sólidos y variados aportes del libro de Juan Carlos brinda una oportunidad excepcional.

Específicamente, presento en este avance tres casos de distinta índole que tuvieron lugar en San Antonio de Areco y permiten iluminar las maneras en que se fueron entablando las articulaciones políticas entre los notables y municipales con el nuevo gobierno durante el Estado de Buenos Aires. En diálogo con los aportes del libro de Juan Carlos reconstruiremos aspectos de la forma en que "vecinos" emprendieron la construcción de un puente sobre el río de Areco, analizaremos las características particulares del presupuesto

\footnotetext{
${ }^{4}$ GARAVAGLIA, Juan Carlos San Antonio de Areco..., cit., pp. 422. El acercamiento al eje planteado estará focalizado en esta ocasión en los notables por limitaciones de espacio y fuentes.

${ }^{5}$ Sobre las municipalidades decimonónicas en Iberoamérica consideramos, entre muchos otros, ESCOBAR OHMSTEDE, Antonio - FALCÓN, Romana y BUVE, Raymond (compiladores) Pueblos, comunidades y municipios frente a los proyectos modernizadores en América Latina, siglo XIX, El Colegio de San Luis, Cedla, México, 2002, pp.245-251; MORELLI, Federica “Orígenes y valores del municipalismo iberoamericano" en Araucania, núm. 18, año 9, 2007, pp. 116-129; MARTINY, Carina "Saber negociar: a inserçao da elite local na estrutura de poder do estado brasileiro (na transiçao do Imperio para a República)", en BESSONE, Tania - BASTOS NEVES, Lucia y GUIMARAES, Lucia (organizadores) Elites, fronteiras e cultura do Império do Brasil, Contra Capa, Río de Janeiro, 2013, pp. 65-87.

6 Los pueblos rurales en Buenos Aires o "pueblos de españoles" según las fuentes, denominación que ocultaba la composición mestiza pero que buscaba diferenciarlos de los pueblos de indios, se fueron conformando a mediados del siglo XVIII por caminos diferentes. Infructuosamente, varios solicitaron ser villas y adquirir las potestades correspondientes, por lo que algunas prerrogativas otorgadas a las municipalidades de mediados del siglo XIX, resultaron inéditas. CANEDO, Mariana "Los "pueblos de españoles" en la monarquía hispánica. La ampliación de jurisdicciones hacia el autogobierno (Buenos Aires, siglo XVIII y primeros años del XIX)", en Prohistoria, núm. 25, Rosario, 2016, pp. 3-27.
} 
presentado por la municipalidad en 1857, y consideraremos algunas tensiones entre jurisdicciones por parte de la municipalidad y el cura párroco, cuya prolongada resolución permitirá vislumbrar cambios más allá del Estado de Buenos Aires en los procesos de negociación y los sujetos intervinientes.

\title{
“El puente de los Martínez" (1854-1857)
}

\begin{abstract}
"En las afueras del pueblo, a unas diez cuadras de la plaza céntrica, el puente viejo tiende su arco sobre el río, uniendo las quintas al campo tranquilo" Ricardo Güiraldes, Don Segundo Sombra ${ }^{7}$
\end{abstract}

Originariamente fue conocido como "el puente de los Martínez", pero pasó a ser el "puente viejo" cuando se construyó otro seis cuadras río abajo. ${ }^{8}$ Ya en el siglo XVIII, Concolocorvo había señalado la conveniencia de un puente sobre el río Areco, al recorrer la zona rumbo al norte minero. ${ }^{9}$ Sin embargo, su construcción está asociada a la familia de notables por excelencia de San Antonio de Areco y al Estado de Buenos Aires.

En el libro, Garavaglia pone de relieve la centralidad del comportamiento de los clanes familiares de los notables de Areco y, particularmente, de los descendientes de Felipe Antonio Martínez. ${ }^{10}$ Las singularidades del caso son señaladas a partir de características de distinto índole. Don Felipe, llegado a Buenos Aires desde Lugo (Galicia) aproximadamente en la década de 1760, con pulperías en la ciudad y en Areco, integró el cabildo de Luján y alcanzó el cargo de comandante de escuadrón. Tuvo nueve y siete hijos con sus sucesivas esposas (ambas pertenecientes a la familia Lima) por lo que no sorprende la densidad de lazos generados en esta pequeña comarca a través de las décadas. ${ }^{11}$ Sin embargo, Juan Carlos particulariza que la red familiar de los Martínez supo integrar a inmigrantes europeos a su intensa alianza de círculos muy restringidos. También, que pese a que dichos comportamientos favorecieron la transmisión patrimonial, algunos de los integrantes de la familia Martínez activaron tramas de relaciones de poder en torno a ese preciado proceso. ${ }^{12}$

Sin embargo, hay otras cuestiones que otorgaron centralidad a los Martínez. Garavaglia señala el prestigio del clan familiar, el destacado papel económico de la familia y las relaciones que fueron estableciendo con el poder. Específicamente, se destaca el lugar medular que la política local (y luego provincial y nacional) ocupó en las preocupaciones de los Martínez, que se tradujo en que la familia "abunda" en alcaldes de la hermandad, jueces de paz, representantes y diputados. Hasta 1880, reconstruye Juan Carlos, hubo nueve miembros de la familia, descendientes directos de Felipe Antonio Martínez, que

\footnotetext{
${ }^{7}$ GÜIRALDES, Ricardo Don Segundo Sombra, EUDEBA, Buenos Aires, 2001, p. 7.

${ }^{8}$ En 1999, el "puente viejo" fue declarado monumento histórico por la Comisión Nacional de Museos y de Monumentos y Lugares Históricos de la República Argentina.

9 BUSTAMANTE, Calixto Carlos Inca Lazarillo de ciegos caminantes desde Buenos Aires, hasta Lima..., Alicante, Biblioteca Virtual Miguel de Cervantes, 2001, capítulo III.

10 Sobre el uso de la categoría "notables", ver particularmente GARAVAGLIA, Juan Carlos San Antonio de Areco..., cit., pp. 139-142.

${ }^{11}$ GARAVAGLIA, Juan Carlos San Antonio de Areco..., cit., pp.152-156.

12 Son múltiples los ejemplos presentados con reconstrucciones, mapas y gráficos en el capítulo IV "Familias y circulación de la tierra" de la segunda parte. GARAVAGLIA, Juan Carlos San Antonio de Areco..., cit., pp.217-251.
} 
fueron jueces de paz; otros fueron electos como municipales e incluso diputados estaduales y provinciales. Norberto de la Riestra fue ministro de Hacienda durante el Estado de Buenos Aires, y luego de las presidencias de Mitre y Avellaneda. De "notables del pueblo de Areco (pasaron) a formar parte de la elite de Buenos Aires y por lo tanto, de la élite de la nación". ${ }^{13}$

Esta permanencia de los Martínez en cargos políticos, observa Juan Carlos, se vio limitada en 1840 tras "la invasión de Lavalle", aunque unos años después algunos de los integrantes de la familia volvieron de su exilio en Montevideo, recuperando sus terrenos embargados -aunque no el ganado(Norberto Martínez en 1844 y José Vicente en 1847). Tras Caseros, los Martínez constituyeron en el pueblo "el poder detrás del trono" durante los meses marcados por la incertidumbre y los enfrentamientos, "prefiriendo no aparecer en primera línea hasta fines de $1853^{\prime \prime} .{ }^{14}$ A partir de allí, su presencia institucional fue tan relevante que es difícil encontrar un expediente relacionado con San Antonio de Areco en el que no surja alguien apellidado Martínez como autoridad o en otras cuestiones de la vida del pueblo. La construcción del puente viejo o de los Martínez, fue una de ellas, como veremos a continuación.

En septiembre de 1854, José Vicente Martínez, “por sí y en representación de algunos vecinos" de San Antonio de Areco, propuso al gobierno del novel Estado de Buenos Aires la construcción de un puente en el río homónimo. ${ }^{15} \mathrm{El}$ mismo se proponía a "cal, canto y madera" o "solo de cal y canto" y estaría ubicado en las afueras del pueblo, como bien describirá Güiraldes en su Segundo Sombra varias décadas después. El gobierno había otorgado ese año la facultad de construir puentes a particulares, uno sobre el río Salado y otro sobre el Tigre en Las Conchas, por lo que el fiscal no encontró objeciones a la petición. Cada caso tuvo sus propias modalidades relacionadas con la configuración social y política en cada partido de la campaña, que serán abordadas en perspectiva comparada en un trabajo específico. En esta ocasión, como adelantamos, el análisis de cómo se promovió y logró realizar el puente sobre el río Areco permite identificar formas de articulación entre los notables del pueblo y el nuevo gobierno a mediados del siglo XIX.

Leemos en el libro de Juan Carlos que en 1854 José Vicente Martínez, de 58 años, era uno de los puntales económicos de la familia y contaba con atributos sociales y políticos destacados en la comunidad. Su representación de "algunos vecinos de San Antonio de Areco", se fue modificando en su expresión a través del expediente. Pedro Bonifacio, el hijo que actuó en su nombre una vez que se aprobó la petición, hablará de los "individuos accionistas". Las autoridades y reparticiones del Estado de Buenos Aires se referirán a "sociedad de empresarios", "los empresarios de San Antonio de Areco", "sociedad de accionistas", "vecinos accionistas", para finalmente en 1857 tender a estabilizarse la denominación como la "Sociedad" o "Comisión del Puente de Areco". No contamos con información sobre la composición de la comisión, aunque José Vicente Martínez, su presidente, Antonio Díaz de Couto, el secretario, y Tiburcio Cano como tesorero, se diferenciaron por haber sido quienes firmaron recibos, se comunicaron con las autoridades y sostuvieron los tres años de trámites y construcción de la obra.

\footnotetext{
${ }^{13}$ GARAVAGLIA, Juan Carlos San Antonio de Areco..., cit., pp. 414-415.

${ }^{14}$ GARAVAGLIA, Juan Carlos San Antonio de Areco..., cit., p. 413.

${ }^{15}$ Archivo General de la Nación (en adelante, AGN), Archivo del Ministerio de Gobierno del Estado de Buenos Aires (en adelante AMGEBA), expediente núm. 7897.
} 
En mayo de 1854, la sanción de la Constitución del Estado de Buenos Aires había declarado la soberanía del mismo, afianzando la separación de la Confederación Argentina. También, se había comenzado a impulsar otra forma de organización institucional, restableciendo experiencias del periodo rivadaviano o fomentando nuevas, a veces partiendo de las existentes. En el proceso de construcción del puente fueron convocados e intervinieron activamente, la Comisión de Obras Públicas (incluyendo la opinión del Departamento Topográfico), ambos en proceso paulatino de "restablecimiento", un fiscal, el ministro de hacienda y el de gobierno y las autoridades locales.

La solicitud de José Vicente Martínez al gobierno de aceptación de la construcción del puente fue presentada con "bases" sobre las que se apoyaba la propuesta. Esta práctica de formulación de "acuerdos" entre particulares y autoridades estaba lejos de constituir una simple formalidad y cada parte incorporó propuestas y cuestionó otras. ${ }^{16}$ En esta oportunidad, mientras la versión inicial de Martínez y los vecinos se centró en establecer disposiciones que hicieran sustentable económicamente la construcción del puente y en asegurar la recuperación de sus acciones, las observaciones de la comisión de Obras Públicas y del fiscal fortalecieron, en diferentes aspectos, la función reguladora del estado. Pedro Bonifacio Martínez, con poder de su padre y tras "conferenciar" con cada uno de los individuos accionistas, aceptó las modificaciones de las bases en abril de 1855, después de siete meses de negociación. Veamos los puntos del acuerdo.

En primer lugar, en la propuesta de Martínez y los vecinos se especificaron las tarifas a cobrar por derecho de pontazgo, una vez terminada la obra. El fiscal, sin entrar a discutir los montos, señaló que no podrían superar lo que "se cobra en los Puentes Propiedad del Estado". Al finalizar el puente, se presentaron tarifas actualizadas a 1857 que fueron aceptadas por el gobierno.

Otra cuestión planteada en las bases fue que, una vez que se reembolsara a la sociedad de accionistas de su principal, con un interés del $6 \%$ anual, se dejaría "al beneficio del Pueblo o municipalidad la obra construida". ${ }^{17}$ El fiscal condicionó el ítem a que la "comisión municipal" de San Antonio de Areco recibiera de los "empresarios" una "razón de lo recaudado" por trimestre. Otro pedido de la sociedad fue que no se construyera otro puente "inmediato" hasta que se hubiesen reembolsado las acciones. Obras Públicas ajustó la propuesta a "una distancia de 2 leguas en ambos lados".

Una sola consideración en las bases propuestas por Martínez y los vecinos incluía a la "autoridad" y lo hacía para garantizar "la solida construcción" del puente. Obras Públicas solicitó un plano del puente y el presupuesto. Señaló modificaciones técnicas al primero que incrementaron al segundo (mayor profundidad en los cimientos, construcción de terraplenes, refuerzo del arco, etc.). En el acuerdo final se especificó que no podría

\footnotetext{
${ }_{16}$ Un ejemplo de la acuerdos negociados entre particulares y autoridades planteados para el bien de la comunidad en estas sociedades ya en el siglo XVIII es el alcanzado entre Francisco de Merlo y el cabildo eclesiástico de Buenos Aires en 1730 por el establecimiento de la sede parroquial en la capilla de Merlo en la campaña a través de siete clausulas. CANEDO, Mariana "Monarquías y élites regionales en torno a oratorios, pueblos de españoles y solares en la primera mitad del siglo XVIII", en CANEDO, Mariana (comp.) Poderes intermedios en la frontera. Buenos Aires, siglos XVIII-XIX, EUDEM, Mar del Plata, 2012, pp. 32-35.

${ }^{17}$ Las municipalidades de campaña fueron instrumentadas a partir de 1856, pero en algunos lugares, como San Antonio de Areco, funcionaron comisiones municipales de manera transitoria y sin demasiada reglamentación específica.
} 
franquearse el "tránsito al público, sin el preciso reconocimiento facultativo de la obra a satisfacción del Gobierno." 18

En febrero de 1856, el plano y el presupuesto para la construcción del puente sobre el río Areco fueron aprobados. El año y medio de duración del tratamiento refleja la dinámica de elaboración de un acuerdo que no tuvo perspectivas encontradas ni demoras particulares.

Un tema central acompañó el acuerdo como consideración específica, sin estar incluido en las bases. Desde la primera propuesta de José Vicente Martínez se solicitó al gobierno que adquiriera "algunas acciones o suministre el auxilio pecuario que tuviese a bien para el fomento de esta empresa". Cuando en febrero de 1855 Pedro Bonifacio Martínez aceptó las modificaciones del acuerdo, pidió al gobierno 40 mil pesos que les faltaban para la obra. En abril, el gobierno anunció que anticiparía la mitad de ese déficits en acciones (40 de \$500 cada una) en "los términos establecidos por la Asociación anónima para la Construcción del mismo puente". Con la obra iniciada, se ordenó al ministro de Hacienda que las efectivizara. ${ }^{19}$

Sin embargo, en 1856, la Comisión del Puente de Areco reconstruyó el proceso de "una obra, puramente en beneficio público" desde el punto de vista de sus finanzas. Se especifica que durante "las tramitaciones" había aumentado el precio de los materiales y que el valor del puente había subido de los $\$ 100.000$ originalmente calculados a $\$ 150.000$. En abril de 1857 , se informaba al gobierno que por la demora de la construcción se habían retirado quince accionistas ( 24 acciones) y se le pedía la suscripción por las acciones que faltaban hasta cubrir el déficit de $\$ 78.500 .{ }^{20}$ Nuevamente, el gobierno accedió parcialmente a partir de 40 acciones más, computadas a Obras Públicas. ${ }^{21}$ Todavía es limitada la información que tenemos sobre el monto final y cómo terminó financiándose la construcción del puente. Según hemos visto, el gobierno participó con $\$ 40.000$, el $27 \%$ del presupuesto de gastos actualizado de la obra y esta fue entregada a la municipalidad de San Antonio de Areco en 1858. ¿Habrá sido suficiente el peaje cobrado en poco más de un año para reembolsar a los vecinos accionistas? Seguramente influyó en el traspaso que en agosto de 1857 las Cámaras del Estado de Buenos Aires aprobaron una ley que establecía que serían "siempre exentos del pago de Contribución Directa, los Capitales invertidos en puentes que se construyan por particulares". También se exceptuaba del pago de "todo arrendamiento, los fundos públicos" sobre los que estos se construyeran. ${ }^{22}$

En junio de 1857, el ingeniero José María Romero, comisionado por el Consejo de Obras Públicas para el reconocimiento del puente, se trasladó a San Antonio de Areco. Allí lo esperaban, si la descripción no responde solo a una

\footnotetext{
${ }^{18}$ AGN, AMGEBA, expediente núm. 7897, folio 137.

${ }^{19}$ AGN, AMGEBA, expediente núm. 7897, folio 194.

${ }^{20}$ El listado de acciones retiradas permite observar quiénes fueron estos accionistas y que la distribución de las acciones fue bastante extensiva: Juan Francisco Font y "Mensajerías argentinas" (4 acciones cada uno), Avelino Isaurralde, Carlos Gómez y Sociedad Hermanos Figueroa ( 2 cada uno); los restantes diez (1 acción por persona). AGN, AMGEBA, expediente núm. 7897, folio 183.

${ }^{21}$ En abril de 1857, los recursos en la obra eran 40 acciones tomadas por el gobierno (\$20.000) y 103 por particulares (\$51.500), lo que llevaba al déficits de $\$ 78.500$. La comisión señalaba los gastos ya pagados que alcanzaban $\$ 107.660 \mathrm{~m} / \mathrm{c}$. AGN, AMGEBA, expediente núm. 7897, folio 189-189 reverso.

${ }^{22}$ AGN, AMGEBA, expediente núm. 14139, Ley del 17 de agosto de 1857.
} 
modalidad, "el Sr. Presidente y Miembros de la Comisión del Puente (...) el Señor Juez de Paz, el Señor Cura Párroco y de varios vecinos". Cabe observar que se mantendría la presencia del cura en este ámbito que en principio podría pensarse ajeno a su especificidad y que mientras el presidente de la comisión era José Vicente Martínez, el juez de paz de ese año (y del anterior) era José Eufemio Martínez, otro de sus hijos (ver gráfico 17 del libro). ${ }^{23}$ En el informe que realizó Romero sobre la obra, pese a que encontró algunas diferencias con el proyecto original y se quejó por la ausencia del constructor encargado por la Comisión del puente, hizo referencias auspiciosas a la obra como: "la más completa y exacta ejecución" con "todas las características de solidez y duración", "la cuota del arco perfectamente brazada, "el conjunto ofrece un aspecto agradable de elegancia". Con el informe, el gobierno, a través del ministro José Barros Pazos, aprobó la obra y comunicó a la sociedad encargada del puente y al juez de paz y presidente de la municipalidad para que "dicho puente sea entregado al servicio público". A casi tres años de la primera solicitud, dos de elaboración del acuerdo y menos de uno de construcción (según la fecha de los recibos por la compra de materiales), comenzaba a funcionar el puente "de los Martínez" en San Antonio de Areco.

\section{El presupuesto de la municipalidad de San Antonio de Areco (1857)}

La línea de investigación que articula fiscalidad y política a partir de los presupuestos se ha consolidado en los últimos años para el Estado de Buenos Aires y la Confederación Argentina a partir de los estudios impulsados por Juan Carlos Garavaglia, posteriores a sus investigaciones con base en San Antonio de Areco. $^{24}$ Sin embargo, el conocimiento sobre la fiscalidad municipal decimonónica es todavía fragmentado, fundamentalmente en los ámbitos rurales. ${ }^{25}$

El establecimiento de las municipalidades en Buenos Aires es señalado por Juan Carlos como el primero de los cambios en la campaña durante el Estado de Buenos Aires. Si bien plantea en 2009 la falta de estudios específicos, señala "el rol dominante que jugará el juez de paz como 'jefe político' de su distrito", al mismo tiempo que reconoce "cierta extensión de los mecanismos locales de poder hacia otros miembros de la notabilidad". ${ }^{26}$

\footnotetext{
${ }^{23}$ El juez de paz era Cecilio Falcón. Descripciones similares fueron realizadas por comisiones del Departamento Topográfico que por esos años recorrían los pueblos de la campaña para realizar sus trazas. En ninguno de los casos aparece una autoridad militar, posiblemente por no tratarse de pueblos de frontera, pero también como particularidad de la formación de los organismos de agrimensores e ingenieros en estas regiones.

${ }^{24}$ GARAVAGLIA, Juan Carlos Construir el estado, inventar la nación. El Río de la Plata, siglos XVIIIXIX, Buenos Aires, Prometeo, 2007; La disputa por la construcción nacional argentina. Buenos Aires, la Confederación y las provincias (1850-1865), Buenos Aires, Prometeo, 2015; GARAVAGLIA, Juan Carlos y CASELLI, Elisa "Guerra, política y negocios en Buenos Aires. Las oficinas de Hacienda y los proveedores del Estado (1858-1860)", en Boletín Del Instituto de Historia Argentina y Americana Dr. Emilio Ravignani, núm. 39, 2013, pp. 39-72.

${ }_{25}$ Algunas excepciones sobre el conocimiento de la fiscalidad municipal mediados del siglo XIX son los trabajos de Marta Bonaudo y Elida Sonzogni para Santa Fe y Viviana Conti y S. Boto para Jujuy, realizados publicados en 1997; el de Inés Sanjurjo para Mendoza para 2004, y la tesis de doctorado de Evangelina de los Ríos sobre Santa Fe de 2013, los de Juan Carlos sobre la municipalidad de la ciudad de Buenos Aires de 2015 y los nuestros sobre las municipalidades rurales de Buenos Aires. Consideraciones sobre estos trabajos se encuentran en CANEDO, Mariana "La política de los presupuestos municipales: conformación de jurisdicciones fiscales en las municipalidades de campaña del Estado de Buenos Aires (1854-1860)", América Latina en la Historia Económica, Instituto Mora, México, en prensa.

${ }^{26}$ GARAVAGLIA, Juan Carlos San Antonio de Areco..., cit., p. 213.
} 
En esta oportunidad, el análisis del primer presupuesto elaborado por la municipalidad de San Antonio de Areco nos permite indagar sobre otra forma de relación entablada entre los notables y el gobierno del Estado de Buenos Aires.

En abril de 1857, el mes indicado por el Reglamento de las Municipalidades de Campaña, el juez de paz y presidente de la municipalidad de Areco, José Eufemio Martínez, presentó al ministro de gobierno, Dalmiro Vélez Sarsfield, el presupuesto para el año siguiente. Era una práctica nueva, iniciada en 1856 con la instalación de las municipalidades de campaña y la asignación por ley de algunos "derechos". Esta cesión de potestades de la jurisdicción del Estado de Buenos Aires a las jurisdicciones municipales, les permitió a los nuevos gobiernos locales elaborar un presupuesto con estimaciones de entradas y gastos, pese a ser considerados insuficientes los derechos a cobrar, aun para las Cámaras. ${ }^{27}$

Una de las características compartidas en las estimaciones de gastos por parte de los municipales fue que superaron ampliamente los ingresos previstos, generando déficits importantes. ${ }^{28}$ En este contexto, analizamos el presupuesto elevado por la municipalidad de Areco en 1857, uno de los pocos cuya estimación de déficits fue bajo, por lo que el gobierno del Estado de Buenos Aires se comprometió a cubrir. En dialogo con el libro de Juan Carlos, buscamos conocer sus características y comprender cómo los municipales lo elaboraron partiendo de rentas limitadas y con numerosos gastos que se presentaban como imprescindibles para el cumplimiento de sus funciones y el "progreso" del pueblo.

Los montos estimados en el presupuesto municipal de San Antonio de Areco resultan en conjunto bajos, aunque relativamente equilibrados: las estimaciones de ingresos corresponden a $\$ 10.100$ y los gastos a $\$ 12.900$ moneda corriente ( $\$ F 510$ y $\$ F 651$ respectivamente, estimados en peso fuerte). Solo hemos ubicado estimaciones presupuestarias más bajas en municipalidades de pueblos de frontera o en proceso de conformación como Patagones o Ensenada (para 1858) y Bahía Blanca (para 1859), distantes de la conformación de Areco constituido desde mediados del siglo XVIII en la zona norte de la campaña. ¿Cómo era San Antonio de Areco en la década de 1850? El análisis sobre la población en la zona de Areco entre 1726 y 1881 presentado en el libro permite avanzar en su caracterización. ${ }^{29}$ Más allá de los ajustes territoriales necesarios en una mirada en el largo plazo, los padrones, censos y otros recuentos de población evidencian que el crecimiento poblacional de la primera mitad del siglo XVIII, característico de la zona norte de la campaña de Buenos Aires, no mantuvo el ritmo en Areco (como sí sucedió en comunidades cercanas como Los Arroyos). Si bien, Juan Carlos observa las limitaciones de fuentes para la población en la segunda mitad del siglo XVIII, los registros que comprenden de 1813 a 1838 fluctúan en torno a las 1.600 personas, cifra no muy alejada de las

\footnotetext{
${ }^{27}$ Los pueblos ("pueblos de españoles" según la documentación del siglo XVIII) no habían logrado prerrogativas fiscales, aunque los jueces de paz participaran en comisiones recaudatorias promovidas por la provincia.

${ }^{28}$ Hemos ubicado 89 presupuestos municipalidades entre 1856 y 1859, de los cuales 82 casos presentaron déficits, cuatro fueron presupuestos equilibrados y en tres casos se estimaron saldos favorables. Entre los 82 presupuestos con déficits, 47 presentaron desequilibrios que alcanzaban a más de $50 \%$ de los gastos. Como contrapartida, hubo nueve presupuestos con déficits con un rango de incidencia relativamente bajo que el gobierno estuvo dispuesto a cubrir. CANEDO, Mariana "La política de los presupuestos...", cit.

${ }^{29}$ GARAVAGLIA, Juan Carlos San Antonio de Areco..., cit., pp. 37-67.
} 
1.266 del año 1744 ni de las 2.030 personas estimadas para 1854. En el Cuadro 2 de la página 58 se sintetiza la información para Areco y su estancamiento incluso descenso- poblacional que contrasta con el crecimiento de la campaña en su conjunto.

En principio, entonces, el presupuesto está pensando para lo que era Areco, una población relativamente pequeña en el contexto del Estado de Buenos Aires. Sin embargo, no todas las pequeñas poblaciones presentaron presupuestos así. ${ }^{30}$ Analicemos las decisiones tomadas por José E. Martínez y los municipales de Areco en la elaboración del presupuesto

\section{Cuadro 1}

Entradas del presupuesto de la municipalidad de San Antonio de Areco (1858)

\begin{tabular}{|l|r|}
\hline "Derecho de Guías & $\$ 6000$ \\
\hline Ídem de corral & $\$ 1200$ \\
\hline Visita de patentes, pesas y medidas en las casas de negocio & $\$ 1000$ \\
\hline Contribución Directa & $\$ 1000$ \\
\hline Delimitación de Solares & $\$ 500$ \\
\hline Extraordinarios" & $\$ 400$ \\
\hline Total & $\$ 10100$ \\
\hline
\end{tabular}

FUENTE: AGN, MGEBA, legajo 13417

El número de ingresos ( 5 de los 10 posibles por el Reglamento de las Municipalidades de Campaña) y la estimación general de entradas fueron moderados en el caso de Areco en términos relativos al grupito de municipalidades con poblaciones similares. ${ }^{31}$ El cobro de las guías a la circulación de productos, los derechos de corrales, o la inspección anual a negocios por patentes, pesas y medidas -que ya se venían cobrando en los diferentes partidos como rentas provinciales-, fueron los impuestos más presentes en los presupuestos de las municipalidades de la campaña y en San Antonio de Areco representaron el $80 \%$ de la estimación de ingresos. En este caso no aparecen presupuestadas multas ni peaje y pontazgo (todavía no estaba habilitado el puente sobre el río Areco y seguramente calcularían cobrarlo los vecinos accionistas). Asimismo, se registró el cobro de 100 pesos a realizar por única vez por la delineación de "edificios" en cada solar, aunque solo estimando cinco casos posiblemente por ser Areco un pueblo ya consolidado en relación a la distribución de los terrenos. No se contemplaron posibles rentas obtenidas de solares de propiedad pública ni el "canon enfitéutico" a cobrar por terrenos públicos de los ejidos de los pueblos, ambos con leyes relacionadas en tratamiento. Sorprende, la baja incidencia calculada por el $10 \%$ de la Contribución Directa, en relación al perfil de los productores que se señalan en el libro (y suponemos que la ley de exención por construcción de puentes todavía no era considerada).

\footnotetext{
${ }^{30}$ Solo Exaltación de la Cruz resulta comparable con Areco en la relación población (2.180 personas) y montos presupuestados para 1858 (\$13000 de ingresos y \$13700 de gastos). Las demás poblaciones similares diseñaron otro tipo de presupuesto que tendió a ser altamente deficitario. AGN, MGEBA, legajo 13159.

${ }^{31}$ La municipalidad de Arrecifes, cercana a Areco y cuyo pueblito fue conformado en la segunda mitad del siglo XVIII, tenía una población de 2795 personas a mediados del siglo XIX. Presentó un perfil de ingresos muy similar (\$10.900), incorporando el cobro de peajes $(\$ 400)$ pero sin considerar la delineación de terrenos. AGN, MGEBA, legajo 13630.
} 
Ahora bien, las decisiones de los municipales resultan más evidentes en la orientación de los gastos.

\section{Cuadro 2}

Gastos del presupuesto de la municipalidad de San Antonio de Areco (1858)

\begin{tabular}{|l|r|}
\hline $\begin{array}{l}\text { “Asignación acordada al Preceptor de la Escuela de varones a 200 p } \\
\text { mensual }\end{array}$ & $\$ 2400$ \\
\hline Salario al encargado del corral a 100 p & $\$ 1200$ \\
\hline Para reparación id del mismo año & $\$ 300$ \\
\hline Compostura de vías públicas id & $\$ 3000$ \\
\hline Reparaciones en la casa del Juzgado y cárcel & $\$ 2000$ \\
\hline Revoque y blanqueo de la Escuela de varones y casa del preceptor" & $\$ 4000$ \\
\hline Total & $\$ 12900$ \\
\hline
\end{tabular}

FUENTE: AGN, MGEBA, legajo 13417

La moderación que caracterizó a los gastos presupuestados para 1858 se centró en proponer reparaciones ( $70 \%$ de lo presupuestado), en vez de construcciones como lo hicieron muchas de las otras municipalidades. ${ }^{32} \mathrm{Al}$ agrupar los gastos por actividades o funciones, se observa que la mitad estuvo orientada a reparaciones de la Escuela, la casa del preceptor y su sueldo. Si bien la educación se encontraba bajo jurisdicción del gobierno del Estado de Buenos Aires que en lo económico se encargaba de pagar los sueldos, alquiler de escuelas, útiles y materiales o premios, varias municipalidades consideraron insuficiente la asignación prevista e incluyeron un "sobresueldo" para complementarla, pese a los cuestionamientos del gobierno. En el caso de Areco, los municipales enunciaron que el monto presupuestado correspondía a la "asignación acordada" y no excluía lo recibido por el gobierno.

El arreglo del corral en el pueblo y un sueldo para su encargado confluyeron en otra de las actividades que orientaron los gastos y que se equilibrarían ese año con los ingresos esperados ante el cobro de los derechos por su uso. Por su parte, la refacción de las calles, representa un monto relativamente importante, que se condice con la descripción que Juan Carlos reproduce por parte de José Ramón Oyuela, prefecto del segundo departamento de la campaña, que expresaba que había que "sacar a este Pueblo de la situación de Aldea en la que se encuentra" por el estado de las veredas y la falta de nombre de las calles, entre otros rasgos, en abril de $1858 .^{33}$

En síntesis, la orientación del presupuesto de gastos presentado hacia la mejora de la educación, el abasto y las calles del pueblo, resultó clara y fundamentada. Ahora bien, José E. Martínez pareciera haber sido consciente de la prudente presentación que realizaba la municipalidad al ministro de gobierno:

\footnotetext{
${ }^{32}$ La comparación con el presupuesto de Arrecifes, recordemos que similar en los ingresos calculados con unos $\$ 10.900$, resulta un ejemplo aunque extremo. La municipalidad de Arrecifes presupuestó gastos por $\$ 365.800$. Ellos se orientaban a la construcción de un puente para el río Arrecifes (\$300.000) y otro para el arroyo que divide el pueblo (\$10.000). Además, consideraron gastos "Para casa de Juzgado, cárcel y cuerpo de guardia" otros $\$ 50.000$ y para el sueldo del secretario y los gastos de escritorio los restantes $\$ 4800$. AGN, MGEBA, legajo 13630. El puente sobre el río de Areco volverá a ser presupuestado por la entonces provincia de Buenos Aires en la década de 1860.

${ }^{33}$ GARAVAGLIA, Juan Carlos San Antonio de Areco..., cit., pp. 417-418.
} 
"Notará VS que no figura en las salidas sueldo al escribiente, alquiler de casa ni gastos de escritorio, por ser servido gratis por uno de las Municipales y tener sus reuniones en la casa del Juzgado; pues siendo tan exiguas las entradas, y tantas las necesidades del Partido se ha procurado hacer todas las economías posibles." 34

En base a este fundamento, José E. Martínez junto con Melquiades Arana, municipal que firmó como secretario interino, solicitaron al "Señor Gobernador" que avalara la decisión de "mandar levantar un plano y presupuesto" para la iglesia que se encontraba "muy deplorable, amenazando ruina" e imploraban su ayuda para la obra. El presupuesto y el aval fueron aprobados, y además, sorprendentemente, Vélez Sarsfield mandó reservar un cargo de "secretario para ser remitido oportunamente a las Honorables Cámaras" a la semana de haber recibido el presupuesto.

En los pueblos de la campaña, los pedidos de ayuda económica para reparar o construir nuevas iglesias brotaron desde 1852, encausados por vecinos y autoridades locales. ${ }^{35} \mathrm{Su}$ resolución fue morosa y, en varias oportunidades, los gastos fueron ingresados en los presupuestos municipales, aumentando consecuentemente los déficits.

La modalidad seguida por la municipalidad de Areco, presidida por José E. Martínez, de presentar un presupuesto moderado pero solicitar gastos específicos por fuera del mismo, potenció los recursos: permitió que el déficits fuese cubierto por el gobierno por no ser excesivo (\$2.800), encausó el arreglo de la iglesia sin incluirlo como gasto e incorporó un secretario cuyo salario podemos estimar entre $\$ 3.600$ y $\$ 4.800$ anuales siguiendo los montos asignados en las otras municipalidades. Cabe señalar que casi todas las otras municipalidades habían solicitado ese empleo y tenido que fundamentar su mantenimiento ante los cuestionamientos del ministro de gobierno.

¿Fue esta forma de proceder en torno al presupuesto resultado de cierta afinidad política entre los municipales y los grupos de poder local con el nuevo gobierno del Estado de Buenos Aires? En principio, no sorprendería que fuera así, aunque todavía falta consolidar con mayor evidencia esta suposición y ampliar la perspectiva comparada, pero sin dudas muestra formas de articulación política entre estos grupos.

\section{Los “terrenos del Santo" (1854-1906)}

El establecimiento de jurisdicciones territoriales por parte de las municipalidades a mediados del siglo XIX fue un proceso más lento, complejo y negociado de lo que se suele considerar. Esto fue así tanto para establecer los límites entre los partidos (jurisdicciones locales) como en la puesta en práctica de potestades sobre la regulación de terrenos al interior de la comunidad local y la especificación de los terrenos públicos. ${ }^{36}$

\footnotetext{
${ }^{34}$ AGN, MGEBA, legajo 13417, folio 2

35 COMAS, Fernanda "La morada de Dios en los pueblos de Buenos Aires. Vecinos y autoridades frente al patronato (1852-1854", en CANEDO, Mariana Poderes intermedios en la frontera..., cit., pp. 181-220.

36 CANEDO, Mariana "Construyendo el territorio tras Caseros. Poderes locales y administradores en el Estado de Buenos Aires (1854)", en Revista Antítesis, Historia Social de la Universidad Estatal de Londrina, Brasil, vol.4, núm.8, 2011; y, "Mucho más que una cuestión de medidas. Las comisiones para el arreglo de los pueblos del Estado de Buenos Aires. Pergamino,
} 
En esta oportunidad proponemos analizar un proceso de negociación en torno a las potestades sobre un terreno en San Antonio de Areco que dura décadas y en el que intervinieron diferentes actores institucionales. El largo proceso permite vislumbrar transformaciones de las formas de establecer acuerdos y de los actores intervinientes durante mediados del siglo XIX y principios del XX.

En julio de 1854, Juan Francisco Font siendo juez de paz de San Antonio de Areco solicitó al gobierno la resolución de una venta correspondiente a un terreno en el ejido del pueblo que consideraba ilegal. ${ }^{37}$ Hacía "unos años" que el cura del lugar, ya fallecido, le había vendido a Don Felipe Figueroa un terreno cuyo origen no resultaba claro. El cura era Feliciano A. Martínez.

A través del libro de Juan Carlos, conocemos a Feliciano Antonio como el integrante de la familia Martínez más comprometido con el rosismo y "personalmente muy cercano a Rosas". ${ }^{38}$ Fue cura párroco de Areco de 1830 a 1844 y junto con Tiburcio Lima (su primo) constituyeron "el vértice nodal del poder local" en esos años. Por su parte, Felipe Figueroa perteneciente a una de las familias con quienes los Martínez se entrecruzaron una y otra vez por casamientos y demás vínculos familiares. ${ }^{39}$ La reconstrucción de los procesos de transmisión y ventas de terrenos de la familia Figueroa, en un círculo estrecho donde los Martínez están presentes, permite conocer la conformación del patrimonio en tierras de Felipe, unas 2.595 hectáreas. ${ }^{40}$ Este proceso mayor emprendido por Felipe Figueroa y otros integrantes de la familia con terrenos en Areco evidencia las modalidades de transmisión empleadas, pero no incluye al terreno cuestionado, ubicado en el ejido como adelantamos.

Ante la presentación del juez de paz en 1854, el gobierno del Estado de Buenos Aires hizo intervenir al fiscal, el Departamento Topográfico y el asesor del ministro de gobierno, quienes vincularon el caso con problemáticas relacionadas con la comunidad en sí. La necesidad de delimitar el ejido del pueblo y el cuestionamiento ante la presencia allí de terrenos particulares, llevó al fiscal a conjeturar la posible "expropiación" del terreno en cuestión. El Departamento Topográfico confirmó que las tierras originariamente "donadas al Pueblo" quedarían al realizarse la traza en gran parte en el ejido, al igual que otros terrenos de propiedad particular. Sin embargo, esta situación no era fundamento para una "expropiación". ${ }^{41}$

Al año siguiente, "el Fiscal" -la documentación no aclara si era una nueva intervención o correspondía a otra persona en el cargo- señaló que según la "Recopilación Castellana" los prelados debían inventariar "las cosas" que recibían o vendían y especificar "el precio". Ante la falta de documentación y de

Arrecifes, San Pedro, 1854-1856" en GARAVAGLIA, Juan Carlos y Pierre. GAUTREAU (editores) Mensurar la tierra, controlar el territorio. América Latina, siglos XVIII y XIX, Prohistoria, Rosario, 2011, pp. 259-294.

${ }^{37}$ AGN, AGEBA, Legajo 7551.

${ }^{38}$ GARAVAGLIA, Juan Carlos San Antonio de Areco..., cit., p. 153 y p. 169.

39 La construcción de reencadenamientos de alianzas entre los Figueroa y los Martínez se describe en GARAVAGLIA, Juan Carlos San Antonio de Areco..., cit., pp. 154-155.

40 Ver particularmente el gráfico 28, "Genealogía de las tierras de Felipe Figueroa en 1857" y la reconstrucción en GARAVAGLIA, Juan Carlos San Antonio de Areco..., cit., pp. 239-244.

${ }^{41}$ Desde el Departamento Topográfico se argumentaba que los ejidos eran considerados como una separación del pastoreo de ganado en una zona reservada para la producción agrícola que podía estar conformado por propiedades particulares como lo estaban mayoritariamente en San Nicolás de los Arroyos y Magdalena. AGN, AMGBA, Legajo 755, folios 249 r y 250. 
prerrogativa por parte del cura Feliciano Martínez para la venta del terreno, el fiscal interpretaba que la operación debía declararse nula.

La intervención del asesor del ministro de gobierno (¿Vélez Sarsfield?) modificó la situación. Si bien coincidió en juzgar, en base al expediente, "nula y de ningún valor la venta que hizo el cura Don Feliciano Antonio Martínez", ubicó al conflicto como de "causa y jurisdicción". ${ }^{42}$ El terreno no era público sino que "se juzga ser de la parroquia" y la resolución competía a los Tribunales ordinarios. A fines de 1855 se dio comunicación a las autoridades que habían intervenido y al obispo diocesano Mariano J. de Escalada.

El expediente iniciado a mediados de 1854 por el juez de paz de Areco finalizó a fines de 1856 con el presidente de la municipalidad solicitando la participación del fiscal general del Estado y el cura de la parroquia nombrando un apoderado, no perteneciente al pueblo. Las formas y los ámbitos de resolución de los conflictos parecieran haber cambiado.

Será en otro expediente, la mensura realizada entre 1905 y 1906, donde a modo de antecedentes aparece más información sobre los caminos seguidos por la venta realizada 60 años atrás. ${ }^{43}$ Allí se transcribieron la merced de tierra que el gobernador y capitán general Don Miguel Salcedo realizó en 1740 al Alcalde Provincial de la Santa Hermandad, Ruiz de Arellano, por sus servicios como súbdito y el prestigio de los antecesores de su esposa, Rosa Giles. También, el poder dado en 1750 por la pareja a Cristóbal Giles, capellán de la capilla de Areco e "hijo adoptivo", para "dar y repartir sitios" dentro de las mil varas de terreno a "personas que quisieran hacer ranchos o casas", dinámica que se vincula a la conformación del pueblo. Asimismo, se reprodujeron 229 ventas que habrían realizado los curas párrocos entre 1750 y 1861, anotadas en el "Libro de toma de la razón de la Iglesia". ${ }^{44}$ Con el número 203 aparece registrada la venta a Felipe Figueroa (con la fecha borroneada y escrito encima el año 1839, aunque el agrimensor señalaba que otra documentación la ubicaba el 24 de febrero de 1840). El objetivo de la mensura fue estimar la superficie total vendida por los curas párrocos de la "donación" realizada por Ruiz de Arellano, para ubicar en ella el terreno cuestionado y discriminar la existencia de tierra fiscal (sobre la cual, recordemos, las municipalidades han adquirido prerrogativas fiscales).

Otro antecedente que se incluye en el expediente por la mensura de principios del siglo XX, es la presentación hecha de la municipalidad de San Antonio de Areco de 1877, ya siendo Buenos Aires una provincia de la República Argentina y Vicente Quesada, ministro de gobierno provincial. A cargo de la municipalidad estaba Isidro B. Martínez.

Se había efectuado la traza del pueblo de San Antonio de Areco que incluía a los terrenos denominados por esos años como "De la Iglesia". La municipalidad señalaba la existencia de un "sobrante propio para chacras" y consultaba si podía venderlo o arrendarlo tras las disposiciones sobre tierra pública sancionadas en $1858 .{ }^{45}$ ¿Era la municipalidad la administradora de todos los terrenos que estaban en el pueblo, incluyendo los "de la Iglesia"? ¿O,

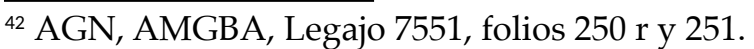

${ }^{43}$ Obras Públicas, Archivo de Geodesia y Catastro, Duplicado de mensura núm. 94, San Antonio de Areco.

${ }^{44}$ Se hacen cuestionamientos a la forma en que se realizaron los registros de ventas. Tres párrocos de la parroquia aparecen comprándose ellos mismos terrenos. Obras Públicas, Archivo de Geodesia y Catastro, Duplicado de mensura núm. 94, San Antonio de Areco, folios, 13 y 13 r. 
era el cura párroco quien debía administrar y cobrar los arrendamientos como él lo había planteado? A estas cuestiones de jurisdicción se le agregaba que el cura, según la municipalidad, impedía el ensanche de los patios de las "Escuelas Públicas" que se encontraban "al lado de los terrenos que sobraban a los fondos del terreno de la Iglesia". ${ }^{46}$ La municipalidad le recordaba al gobierno provincial los "grandes sacrificios" realizados por el Superior Gobierno y el vecindario para formar los "Edificios públicos" ("refacción completa del Templo, Casa del Señor Cura y últimamente la traza y delineación del pueblo") y remarcaba la importancia de la decisión que le solicitaba. El argumento, expresado a fines de la década de 1870, se acerca más a las solicitudes a una autoridad de antiguo régimen cuya función como juez era mediar, otorgar a cada uno lo que le correspondía, ponderando los "sacrificios" realizados, más que esperar que se procediera en base a las leyes sancionadas. ${ }^{47}$

Con antelación a la posible respuesta, el apoderado del cura párroco y la municipalidad de Areco presentaron un acuerdo al gobierno provincial. Desconocemos todavía las tramas que llevaron a que se efectuara, pero destacamos que en él se proponía que, por un lado, la municipalidad reconociera a la Iglesia como propietaria del terreno denominado El Santo y su posesión tranquila y no interrumpida desde 1750, tanto como la administración directa y exclusiva de los terrenos al cura. Por el otro, "la Iglesia de San Antonio de Areco" cedía en beneficio de la municipalidad una superficie entre las 6 y 10 cuadras (a precisar tras la mensura) por el "progreso y las conveniencias del Partido" y "el engrandecimiento y mejoramiento moral y material de la feligresía". La municipalidad reservaría tres de las cuadras cedidas para "plaza, escuelas, hospital, cementerio o algún establecimiento o institución de interés público, no para objetos y propósitos particulares". ${ }^{48}$

Ese primer acuerdo fue modificado en varias oportunidades y en el expediente se encuentran informes del asesor del gobierno y de representantes de la iglesia, nuevas bases consensuadas y más cuestionamientos. Estos últimos se centraron, por un lado, en modificar la cesión de los terrenos planteada por el apoderado del cura hacia la municipalidad (de 6 a 10 cuadras) y, por otro, en cuestionar la falta de títulos por parte de la iglesia y sus derechos sobre los terrenos en disputa.

El conflicto por prerrogativas y derechos sobre la compra-venta de un terreno pasó a ser encuadrado como tensiones de jurisdicciones entre la municipalidad y la iglesia en San Antonio de Areco, mientras la formación del estado nación y de la iglesia nacional iban tomando cuerpo. ${ }^{49}$ En el proceso se fueron esbozando algunos indicios de transformaciones que en términos del

\footnotetext{
${ }^{45}$ BARCOS, Fernanda Pueblos y ejidos de la campaña bonaerense. Una historia socio-jurídica de los derechos de propiedad y la conformación de un partido: Mercedes, 1780-1870, Prohistoria, Rosario, 2013.

${ }^{46}$ Obras Públicas, Archivo de Geodesia y Catastro, Duplicado de mensura núm. 94, San Antonio de Areco, folios 16 y $16 \mathrm{r}$.

${ }^{47}$ Las reflexiones en este sentido en las jurisdicciones locales, según los cambios de organización de gobernó local, puede verse en el capítulo "La cruz, la vara y la espada". GARAVAGLIA, Juan Carlos San Antonio de Areco..., cit., pp. 167-194.

${ }^{48}$ Obras Públicas, Archivo de Geodesia y Catastro, Duplicado de mensura núm. 94, San Antonio de Areco, folio 23r.
}

${ }^{49}$ DI STEFANO, Roberto "Sobre liberalismo y religión: rentas eclesiásticas y presupuesto de culto en el Estado de Buenos Aires (1852-1862), en Almanack, núm. 5, 2013, pp. 178-197. 
libro de Juan Carlos podrían ser considerados en camino hacia la "modernidad" argentina. Uno es a nivel de los sujetos que actuaron en la resolución del conflicto. Por un lado, la municipalidad pareciera haberse consolidado como cuerpo y persona jurídica, aunque buscó ser representada por el asesor del gobierno provincial para consolidar sus prerrogativas en el ámbito de su territorio ante la intervención de la iglesia. El cura párroco aparece en los expedientes siendo desplazado paulatinamente en su protagonismo. De los curas párrocos vendedores (y compradores) de terrenos en Areco, incluyendo el cuestionado Feliciano Martínez, al cura de la década de 1870 que acude a un representante por fuera de la comunidad, para finalmente quedar desdibujada su figura por la de la Iglesia como actor en el ámbito provincial o nacional.

Otro indicio de transformaciones resulta ser que las peticiones impactaban en la comunidad de San Antonio de Areco (el terreno del ejido que se podía arrendar o vender, o la ampliación del fondo de las escuelas), pero su resolución no se restringía a su ámbito. Las instituciones provinciales del gobierno, el fiscal y la curia eclesiásticos, la Suprema Corte de la provincia, entre otros, se conformaron como sujetos intervinientes en la propia trama de los reclamos. Los procesos mediadores persisten y se ponen de manifiesto en la documentación consultada. Sin embargo, los espacios y sujetos políticos de negociación parecieran estar jerárquicamente dispuestos.

Nuevamente, como Juan Carlos llama la atención oportunamente, es necesario considerar los cambios de la documentación y la forma en que la información se presentaba en ella hacia fines del siglo XIX y principios del XX. Las instituciones y los cargos de las autoridades eclipsan a los sujetos nominales y los entramados familiares, por lo que la búsqueda en otro tipo de documentación que la recupere permitirá mejorar la comprensión sobre la profundidad de las transformaciones de estas prácticas. ¿Se mantuvo, acaso, un entramado local que impulsaba, resistía o trababa la resolución de este largo proceso?

A su vez, vuelve a ser necesario discriminar los lenguajes utilizados y sentidos otorgados en cada periodo. Por ejemplo, el "terreno de Ruiz de Arellano" sobre el que se otorgó "poder" de una fracción pasó a ser terreno denominado como "de la Iglesia" en la segunda mitad del siglo XIX y "Del Santo" a principios del siglo XX (el Departamento Topográfico refiere a "del pueblo" en 1854). Pero además las ventas registradas por los propios curas fueron presentadas bajo el título de "Desmembramiento del terreno de El Santo" en el siglo XX, afianzando la idea de terreno unido y con esa identidad.

\section{A modo de cierre}

Conocí a Juan Carlos cuando iniciaba sus investigaciones sobre San Antonio de Areco. Cada semana reflexionaba sobre los pastores y labradores del siglo XVIII en el primer seminario que dictó sobre la problemática de la fuerza de trabajo en la campaña de Buenos Aires. Todavía recuerdo el entusiasmo con que nos mostró, a quienes concurríamos a Tandil para cursar con él, un cuadro que ponía de relieve la importancia proporcional de quienes ocupaban la tierra en Areco, sin título de propiedad, poniéndola en valor con fuerza de trabajo fundamentalmente familiar. Señalaba, por entonces, la importancia de los padrones del siglo XVIII como fuente que, prácticamente, no había sido consultada para Buenos Aires y nos impulsaba a emprender su análisis en nuestros trabajos en curso (de hecho, buena parte de mi tesis de licenciatura en 
la UNICEN y algún capítulo de la tesis de doctorado en la UBA se estructuraron en base a ellos). También compartía por entonces, casi sorprendido, la desproporción temporal que existía entre el proceso que le había permitido llegar al cuadro y su presentación en la clase, pese a que la realizaba con todo su histrionismo logrando hacernos sentir parte de la investigación. Con todo, la información del cuadro, central para comprender las relaciones sociales entabladas en la campaña, lo acompañó en diferentes trabajos empezando por el desafiante "¿Existieron los gauchos?" que formó parte del Debate publicado en el Anuario 2 del IEHS y también, de alguna manera, se encuentra incluida en San Antonio de Areco, 1680-1880. ${ }^{50}$

A 30 años de esos recuerdos, me encuentro por primera vez escribiendo sobre San Antonio de Areco, dialogando con las ideas y conocimiento plasmados por Juan Carlos en el libro. Los casos seleccionados permitieron considerar algunas formas de articulación entre los notables y municipales con el gobierno del Estado de Buenos Aires, considerando la configuración del poder local en Areco. En primer lugar, analizar la política en San Antonio de Areco a mediados del siglo XIX es ratificar lo planteado en Juan Carlos sobre la importancia de la familia Martínez, fundamentalmente desde 1854. No es este el único caso de familias de notables que se destaquen por las articulaciones políticas con los gobiernos en Buenos Aires y, pese a las distancias, también las élites provinciales tuvieron algunas características comparables. Sin embargo, la particularidad de los Martínez pareciera estar en la intensidad alcanzada a partir de conformarse como clan de una relativamente pequeña comunidad rural y su presencia casi dominante en los emprendimientos realizados en ella. La expresión de la cantidad de cargos ocupados de manera casi continua en la política sobre todo local, pero también liderando comisiones $u$ otras modalidades, y el entramado de la familia activado en ellos, son manifestaciones de esta característica.

A través de las situaciones analizadas se puso en evidencia la importancia otorgada a los acuerdos formalizados entre particulares y autoridades sobre cuestiones vinculadas al bien común o público de la comunidad. Si bien resulta una práctica ya conocida en la campaña, resultó una forma de articulación central, como vimos, en el proceso exitoso de construcción de un puente en el río de Areco a mediados del siglo XIX. José Vicente Martínez y su hijo, junto a los vecinos accionistas, estuvieron dos de los tres años que demandó el emprendimiento estableciendo las bases del acuerdo con autoridades y departamentos del Estado de Buenos Aires. Pudimos reconstruir cómo los objetivos de sustentabilidad económica y buena construcción de la obra fueron logrados a partir de los mutuos ajustes a la propuesta presentada por los vecinos, aparentemente sin disenso entre las partes.

En todo el emprendimiento, la distinción de actividades parece haber sido complementaria. José Vicente Martínez y los vecinos accionistas propusieron la construcción del puente, una modalidad para emprenderlo y sustentar su construcción, acordaron las modificaciones a las bases propuestas, compraron el material y encargaron a un constructor la realización de la obra. Se vieron beneficiados por lo que significaba un puente para la comuna de Areco, posiblemente varios de ellos además como productores agrarios y comerciantes del lugar, y por la exención de la Contribución Directa sancionada

\footnotetext{
${ }^{50}$ GARAVAGLIA, Juan Carlos “¿Existieron los gauchos?”, en Anuario del IEHS, núm. 2, 1998, pp.42-52.
} 
por las Cámaras. Por otra parte, fueron varias las autoridades y los departamentos que intervinieron en cuestiones especificas: regularon técnicamente la construcción al inicio y final de la obra; ajustaron el accionar de la sociedad de vecinos accionistas; otorgaron ayuda económica (aunque parcial y cuando la obra ya había empezado); establecieron, primeramente, potestad a los vecinos accionistas en el cobro del pontazgo y luego sancionaron la ley de exención de la Contribución Directa ya mencionada. Podemos suponer que esta modalidad de emprendimiento con participación de vecinos accionistas resultó beneficiosa para el gobierno en la distribución de los gastos en una coyuntura compleja del Estado de Buenos Aires.

La búsqueda de acuerdos formales entre la municipalidad y la iglesia de Areco también aparece como una práctica valorada durante la segunda mitad del siglo XIX, pero la posibilidad de lograr consensos por la jurisdicción de terrenos en el pueblo presentó reiteradas dificultades. El expediente, iniciado por el juez de paz en 1854, cuestionaba la venta de un terreno por parte del cura Felipe A. Martínez en 1840, pero los diferentes encuadres propuestos por el fiscal, el Departamento Topográfico y el asesor del ministro de gobierno evidencian que se estaban produciendo cambios o precisiones en torno a lo público y lo particular, y específicamente en torno a la iglesia como sujeto. Estas cuestiones, junto con el fortalecimiento de las municipalidades y la consolidación del proceso de construcción del estado nación y la iglesia como institución y sujeto político, continúan vertebrando el expediente en las siguientes décadas del siglo XIX.

Aunque la disputa se refiere a jurisdicciones sobre terrenos de la localidad, la resolución y sus implicancias parecieran estar cada vez más alejadas de Areco. Los tipos de representación del cura y la municipalidad por fuera de lo local, los acuerdos presentados pero posteriormente modificados por ellos mismos, los cuestionamientos y apelaciones que reciben por parte de instituciones o autoridades diferencias a nivel provincial que dificultan la aprobación de acuerdos, muestran tramas más jerarquizadas con el correr del siglo XIX.

Sin embargo, como Juan Carlos señalaba en el libro y no parece un tema menor, las características de las fuentes han cambiando. Tanto en el proceso de construcción del puente como en el reclamo por el terreno, las diferentes denominaciones de los vecinos o del terreno parecieran exteriorizar cambios en el sentido otorgado a cada caso. A su vez, el protagonismo de las instituciones desplazando a las personas, oscurece la reconstrucción y deja interrogantes sobre si otras tramas continúan, ahora más eclipsadas en las fuentes.

Por último, otra forma de lograr acuerdos, aunque en esta ocasión como una práctica más flexible y puntual, se puso de manifiesto a partir de analizar las particularidades del presupuesto presentado por la municipalidad de Areco, presidida por José Bonifacio Martínez. En un contexto donde las municipalidades presentaron presupuestos estimados con déficits relativamente importantes, los municipales de Areco tuvieron prácticas que potenciaron sus recursos. Elaboraron un presupuesto moderado, relativamente equilibrado y plausible de ser cubierto por el gobierno, que concentraba los gastos para educación, el abasto, y mejoramiento de las veredas y calles. Los gastos mayores como la construcción de la iglesia (o como lo fue el puente), no fueron incorporados al presupuesto como había sucedido en otros casos. Asimismo la no solicitud de un secretario, recurrentemente pedido en el resto de las municipalidades y cuestionado por el gobierno, fue otorgado para Areco por el 
ministro. En conjunto, los municipales de Areco lograron potenciar sus recursos, y encontraron un gobierno muy receptivo antes sus decisiones, lo que nos permite suponer cierta afinidad política a seguir profundizando.

Como fuimos expresando, el libro de Juan Carlos nos permitió otorgar mayor profundidad a los documentos e intentar comprender mejor las tramas analizadas, actividad difícil de replicar con otras municipalidades por falta de esfuerzos similares. Quiero creer que Juan Carlos se hubiese entusiasmado con las situaciones presentadas, como lo hacía con los trabajos que leía. Seguramente hubiese hecho observaciones, aportado información y nuevas preguntas para seguir indagando. Cada lectura a sus libros y artículos siguen transmitiendo ese entusiasmo tan particular por el conocimiento, sus reflexiones y compartiendo desafíos. El dialogo, en parte, continúa.

Mar del Plata, 24 de noviembre de 2017 